\title{
Impacts of a Comprehensive TB Control Model on the Quality of Clinical Services and the Financial Burden of Treatment for Patients with Drug- resistant Tuberculosis in China: A Mixed-methods Evaluation
}

Weixi Jiang

Duke Kunshan University https://orcid.org/0000-0002-5643-6255

\section{Zhipeng Li}

School of Public Health, Fudan University

Qi Zhao

School of Public Health, Fudan University

Mengqiu Gao

Beijing Chest Hospital, Capital Medical University

Qian Long

Global Health Research Center, Duke Kunshan University

Weibing Wang

School of Public Health, Fudan University

Fei Huang

National Center for Tuberculosis Control and Prevention, China CDC

Ni Wang

National Center for Tuberculosis Control and Prevention, China CDC

Shenglan Tang ( $\sim$ shenglan.tang@duke.edu )

Duke University https://orcid.org/0000-0001-9214-7787

\section{Research Article}

Keywords: drug-resistant tuberculosis, quality of health services, financial burden

Posted Date: December 15th, 2020

DOI: https://doi.org/10.21203/rs.3.rs-126542/v1

License: (c) (1) This work is licensed under a Creative Commons Attribution 4.0 International License.

Read Full License 
Version of Record: A version of this preprint was published at Infectious Diseases of Poverty on April 21st, 2021. See the published version at https://doi.org/10.1186/s40249-021-00832-5. 


\section{Abstract}

\section{Introduction}

Drug-resistant tuberculosis (DRTB) has become a critical challenge to ending TB efforts worldwide. To help address the burden in China, the China National Health Commission-Gates TB Project Phase III implemented a comprehensive TB control model which includes multiple interventions to improve the quality of clinical services and reduce the financial burden for DRTB patients.

\section{Methods}

A mixed-methods approach was used to evaluate the effectiveness of interventions. The quantitative data included three sources: de-identified DRTB registered data from 2015-2018 in project provinces, medical records of DRTB patients registered in $2018(n=106)$, and a structured DRTB patient survey in six sample prefectures. The quality of clinical services was evaluated using seven indicators across patient screening, diagnosis and treatment. Logistic regression was conducted to explore factors associated with the extremely high financial burden (defined as out-of-pocket medical expenditure in the first six months of treatment over $30 \%$ of annual household income). Key informant interviews with policymakers and focus group discussions with physicians and DRTB patients were conducted to understand the interventions implemented and their impacts.

\section{Results}

The percentage of bacterially confirmed patients taking a drug susceptibility test (DST) increased significantly between 2015 and 2018: from $57.4 \%$ to $93.6 \%$ in Zhejiang, $12.5 \%$ to $86.5 \%$ in Jilin, and $29.7 \%$ to $91.4 \%$ in Ningxia. The treatment enrollment rate among diagnosed DRTB patients also increased significantly and varied from $73 \%$ to $82 \%$ in the three provinces in 2018 . Over $90 \%$ of patients in Zhejiang and Jilin and 75\% in Ningxia remained in treatment by the end of the first six months' treatment. Among all survey respondents $77.5 \%$ incurred extremely high financial burden of treatment. Qualitative results showed that interventions on promoting rapid DST technologies and patient referral were successfully implemented, but the new financing policies for reducing patients' financial burden were not implemented as planned.

\section{Conclusions}

The quality of DRTB related clinical services has been significantly improved following the comprehensive interventions, while the financial burden of DRTB patients remains high due to the delay in implementing financing policies. Stronger political commitment and leadership are required for multichannel financing to provide additional financial support to DRTB patients.

\section{Introduction}


Drug-resistant tuberculosis (DRTB) presents a new challenge to bringing an end to TB globally [1]. According to the World Health Organization (WHO), 3.3\% of new TB patients and $18 \%$ of previously treated patients developed rifampicin-resistant TB (RRTB) in 2019, including multi-drug resistant TB (MDRTB). China has a high burden of DRTB: it made up 14\% of the total RR/MDR-TB cases in 2019. China together with India also accounted for $41 \%$ of global gaps between the number of RR/MDR-TB patients enrolled in treatment and the estimated number of new cases as reported by the WHO [2]. There is an urgent need for effective DRTB control strategies and policies to achieve the Sustainable Development Goal of ending the TB epidemic by 2030 in China [3].

Previous studies in many low-and-middle income countries, including China, have revealed several barriers to improving case detection and treatment of DRTB. The diagnosis of DRTB takes around two months using traditional technologies, such as a culture test. The long waiting time for diagnosis has largely contributed to pre-diagnosis dropout of patients, especially for poor patients who exhaust available funds during pre-diagnosis treatment [4-6]. Besides, the standard treatment for RR/MDR-TB patients is expensive and time-consuming, and the accessibility of quality treatment has been challenging in developing countries [7, 8]. In China the standard treatment takes 18 months, including both hospitalization and monthly outpatient visits after being discharged from the hospital. However, the financial protection provided by the three types of public health insurance in China, [the urban employee basic medical insurance (UEBMI), urban resident basic medical insurance (URBMI), and new rural cooperative medical schemes (NCMS)], is often insufficient to cover the costs. One major reason is that many medical services, such as some second-line anti-TB drugs, are only partially covered by insurance or may need to be paid fully out-of-pocket [9-13]. Additionally, under China's current TB control model, DRTB patients are treated in the prefecture-level designated hospital. These facilities are typically a thirdlevel hospital with relative low reimbursement rates for patients with either URBMI or NCMS. Therefore, the standard treatment is often unaffordable to many DRTB patients and consequently, patients either default treatment or sell their properties or borrow money to cope with the treatment cost, and become poverty-stricken [14-17]. Poor adherence to treatment may also influence the treatment outcome. According to the WHO, the treatment success rate for RR/MDR-TB patients in China who started on second-line treatment was $54 \%$ in 2017 [18].

A number of interventions have proven effective in providing high quality clinical services to presumptive and diagnosed DRTB patients. Rapid drug susceptibility test (DST) technologies, such as GeneXpert, can provide DST result for rifampicin in a single day. Rapid DST has been proved effective in improving case detection, thereby reducing the likelihood of pre-diagnosis attrition [19-22]. Other interventions focusing on the management of DRTB patients, such as directly observed therapy, patient-centered support, and the use of e-health technologies, could also improve treatment adherence of MDRTB patients [23, 24]. The evidence on the effectiveness of providing additional financial support on the accessibility and outcomes of DRTB treatment is relatively scarce, though many international donor-sponsored TB control projects involve economic support for patients [25]. Nevertheless, one comprehensive program providing universal access to health care for MDRTB patients improved the treatment initiation rate and adherence to treatment, and significantly decreased out-of-pocket (OOP) medical expenditures [26]. Another study also 
found that psychological and financial support provided together could improve the cure rates for MDRTB patients [27].

To address TB control challenges in China, the China National Health Commission (NHC)-Gates TB Project Phase III has implemented a new comprehensive model of TB control in three Provinces: Zhejiang, Jilin, and the Ningxia Hui Autonomous Region (herein referred to as ZJ, JL and NX). The China National Health Commission- Gates TB project has been developing and implementing an innovative TB prevention control model since 2009. Phase I focused on MDR-TB case finding and treatment, and Phase II piloted a comprehensive approach including health system strengthening, creative financial mechanisms, and the use of new technologies in three prefectures [28]. Based on experiences from previous phases as well as other scientific evidences, the model for Phase III included a set of interventions aimed at 1) improving the accessibility and quality of DRTB-related health services and 2) relieving the financial burden of DRTB patients. According to the intervention design, rapid test facility such as GeneXpert would be equipped at both the prefecture and county-level TB designated hospitals for DRTB screening, and the supply of reagents would also be guaranteed through supporting policies. Besides, the bi-directional referral policies require that the presumptive DRTB patients be transferred to the prefecture-level hospital for diagnosis and standard treatment. Multiple financing resources, including both public health insurance and government specialized funding, would also be mobilized to provide additional financial protection for DRTB patients and reduce the percentage of out-of-pocket (OOP) medical expenditures to $10 \%$. As part of the project evaluation, this study aims to assess whether the designed interventions were successfully implemented in the project provinces and investigate the impacts of these interventions on the clinical performance of DRTB related health care services in terms of case detection and treatment, and the financial burden of DRTB patients during the intervention period.

\section{Methods}

\section{Study sites}

The study was conducted in the three project provinces where the Gates TB Project Phase III was implemented: $\mathrm{ZJ}, \mathrm{JL}$, and $\mathrm{NX}$, and these sites were selected according to their range in socioeconomic development in order to examine the effects of interventions in different resource settings. $Z \mathrm{~J}$ is the most developed province in eastern China with an annual disposable per capita income of CNY 45,840, ranking $3^{\text {rd }}$ among the 31 provinces in 2018. $\mathrm{JL}$ and NX are less developed than ZJ, ranking $18^{\text {th }}$ and $22^{\text {nd }}$ respectively [29]. Under the TB prevention control model in ZJ and JL, the anti-DRTB treatment should be provided by the prefecture-level designated medical institutions, usually comprehensive third-level hospitals in ZJ and specialized infectious disease hospitals or TB dispensaries in JL. In NX only the provincial level hospital, an infectious disease hospital, provide standard anti-DRTB treatment. In addition to the data collection at the provincial level, we also conducted in-depth evaluations in six sample prefectures, two in each province. In each province, we selected one prefecture with a relatively high level of economic development and one with a lower level of economic development. 


\section{Study design}

This study used a mixed-methods approach. We combine quantitative analyses of DRTB case records from the Tuberculosis Information Management System (TBIMS), medical records from DRTB designated hospitals, and data from a DRTB patient survey with qualitative research with key informants on the clinical service for and financial burden of DRTB.

\section{Data collection}

\section{TBIMS records and medical records}

The de-identified dataset of DRTB patients registered between 2015 and 2018 in ZJ, JL and NX was retrieved from the TBIMS of China Center for Disease Control (CDC). While previously only MDR/XDR-TB patients were registered as a specialized category in TBIMS, in recent years patients resistant to at least one type of first-line anti-TB drug are gradually registered and included in standardized anti-DRTB treatment according to their DST results. The dataset contained three basic sets of information: 1) demographic information of DRTB patients including age, gender, ethnicity, residence, etc.; 2) diagnosis and treatment information, including all drug-resistant tests (DST) taken, the results, and the status of any anti-DRTB treatment; and 3) sputum and culture tests taken during the treatment period.

The medical records of all DRTB patients registered in 2018 in the six sample prefectures who had completed inpatient treatment were retrieved from designated hospital. In total 106 medical records were examined: 24 in ZJ, 58 in JL, and 22 in NX. These records were examined by clinical experts according to the following constraints: 1 ) the usage of molecular DST;2) the rationality of treatment regimen, defined as whether drugs in the prescription were selected based on DST results using the guidelines developed by China CDC; and 3) the treatment status and the negative rate of culture test after 6-months' treatment. Similar examination was also conducted at the baseline survey in 2015 , but the sample size $(<50)$ was too small for the comparison before and after intervention.

\section{DRTB patient survey.}

The DRTB patient survey was conducted in the six sample prefectures in August 2019. The questionnaire collected information on patient demographic and socio-economic status, treatment experiences, treatment costs, and OOP expenditures during the first six months of treatment. Eligible participants include DRTB patients that were registered between February 1, 2017 and February 1, 2019 in the six study sites who had completed six months of treatment. Patients were sampled successively following a reverse chronological order of the registration date. The expected sample for each prefecture was 70 . If the total number of eligible patients was less than 70 , then all patients were sampled. Face-to-face 
interviews were conducted by trained investigators using a structured survey questionnaire loaded in a tablet PC.

\section{Qualitative data}

Semi-structured in-depth interviews were conducted to understand the how the designed interventions were implemented during the project period. In-depth interviews were conducted with informants from provincial and prefectural level health commission $(n=9), \operatorname{CDC}(n=9)$, health insurance office $(n=9)$ and the insurance division of designated hospital of the sample prefectures $(n=9)$.

Focus group discussions were conducted to understand patient treatment experiences and causes of financial burden. FGD were held with physicians in designated hospitals $(n=7)$ and DRTB patients $(n=6)$, and each focus group had 4-6 participants. Eligible DRTB patients were those who had completed 6 months of treatment. Patients were selected based on their gender, socio-economic status, insurance type, and distance from downtown areas.

All interviews and FGDs were conducted in a private room by experienced researchers. Oral consent was obtained before tape-recording. On average, each interview lasted 30-45 minutes and the FGD was around one hour.

\section{Data analysis}

The quality of clinical service for DRTB patients was evaluated in the three areas: 1) patient screening and diagnosis, including the percentage of bacteriologically positive TB patients ever taking DST and molecular DST; 2) treatment status, including the percentage of patients included in treatment and remain under treatment six months after treatment initiation; 3 ) rationality and outcome of treatment, including the percentage of patients taking the culture test, the percentage of patients with negative culture test results after six months of treatment, and the proportion of rational regimen (table 1). Indicators a-f were calculated using the TBIMS records for patients in 2015 as the baseline, and in 2018 as the final evaluation. Chi-square tests were conducted to see if there were significant differences between the baseline and the final. Data from the examination of the medical records were also used to calculate indicators $\mathrm{d}-\mathrm{g}$. Results from the two resources on the same indicator would also be crosschecked for validation, and were reported by province. 
Table 1

Key indicators for evaluating the quality of clinical service for DRTB patients

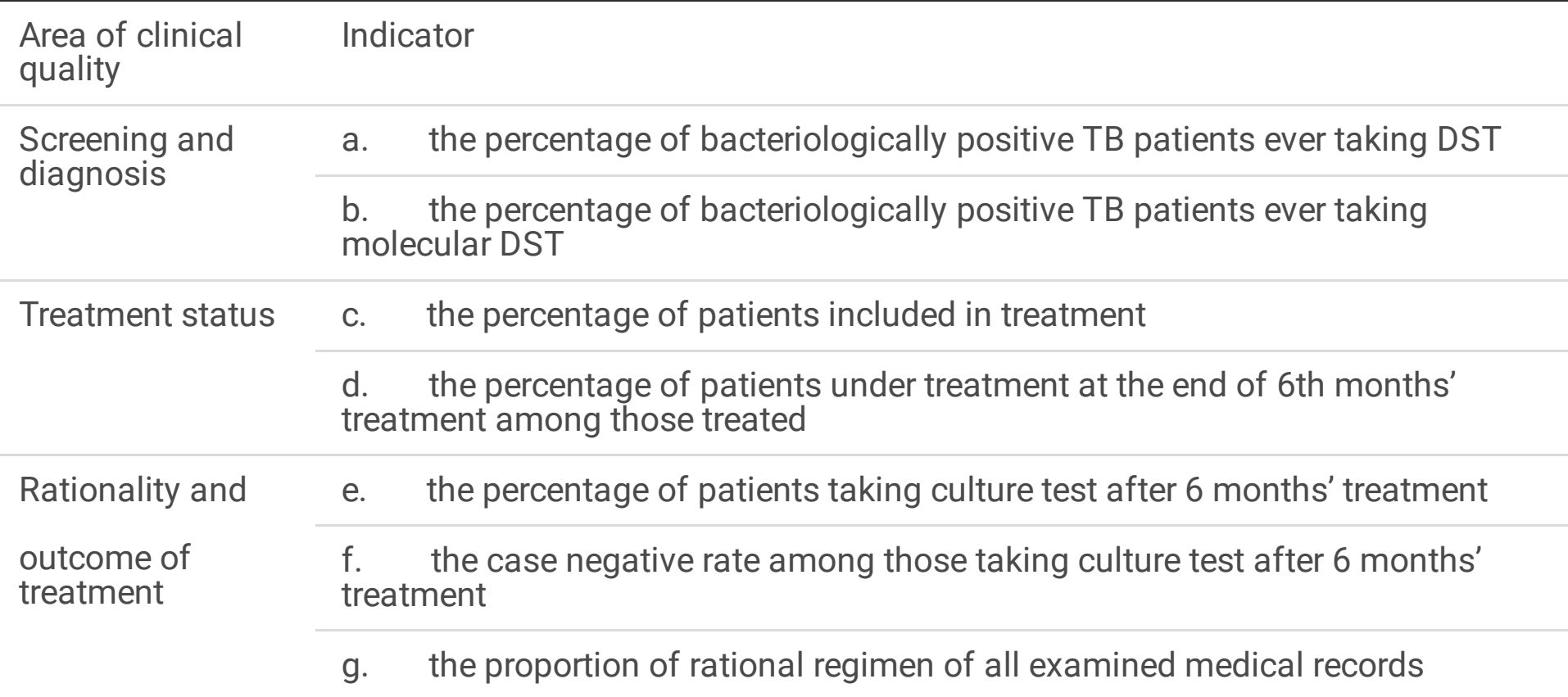

For the patient survey, descriptive analysis was conducted to understand basic patient characteristics. Among the 204 respondents of the survey, 147 (72.1\%) reported OOP medical expenditures. Therefore, we compared the characteristics of those patients who reported OOP expenditures with those who did not using a chi-squared test. A multiple imputation (MI) method was used to address missing data in the selfreported OOP medical expenditures, and the analysis of the financial burden were based on the results of MI [30]. Self-reported OOP medical expenditures were calculated for the first six months of treatment. The financial burden of patients was evaluated using the three thresholds of incurring catastrophic medical expenditure, defined as OOP medical expenditure exceeding 10\%, 20\% and 30\% of annual household income respectively [31]. Logistic regression was conducted to explore factors associated with the extremely high financial burden of treatment (defined as OOP medical expenditure $>=30 \%$ of annual household income).

Qualitative data was analyzed using a thematic analysis approach [32]. The analytic framework was developed based on the topic guides, and was refined regarding the emerging themes during the coding process. All qualitative data were coded and classified under each theme of the analytic framework, and we compared findings by different stakeholders to identify commonality and divergent perceptions. We analyzed the original Chinese texts and translated the results to English.

\section{Results}

\section{Changes in the quality of clinical services}




\section{1) Screening and diagnosis}

DRTB patient records in TBIMS show that the number of diagnosed patients increased drastically from 766 in 2015 to 1765 in 2018. Specifically, the number of diagnosed DRTB patients increased six-fold in $\mathrm{JL}$, from 141 in 2015 to 1016 in 2018. Compared to 2015, DRTB patients in 2018 tended to be older in age, and there were higher percentage of patients living in the county of registered residence. Among all registered patients, about $73 \%$ were male, and the ethnicity other than Han was primarily the Hui Nationality in NX (see supplemental table 1)

Results from the TBIMS data show that the percentage of bacterially confirmed patients taking DST increased significantly in all three project provinces (Figure 1). Prior to project implementation in 2015, the proportion was only $12.5 \%$ in $\mathrm{JL}, 29.7 \%$ in NX, and $57.4 \%$ in ZJ. Despite varying performance prior to the project, by 2018 , the proportion in each province was $86.5 \%, 91.4 \%$, and $93.6 \%$ respectively. The proportions of bacterially confirmed patients taking molecular DST among those tested also increased from less than $50 \%$ at baseline in all three provinces, to $79.8 \%$ in Z J, 78.8\% in $\mathrm{JL}$ and $90.0 \%$ in NX (see supplemental table 2 for detailed figures and results of chi-square tests).

Qualitative studies revealed that successful scale-up of the new rapid DST technologies, mainly GeneXpert, contributed to the increase of DR-TB case finding. Most key informants indicated that the successful promotion of the new DST technologies relied on government funding to guarantee the reagents for the assays and provide the screening test free-of-charge for presumptive DRTB patients, thus ensuring the use of the rapid DST technologies when necessary. Nevertheless, some physicians expressed concern that the funding support for reagents could not be sustained after the end of project. As a way to mitigate such sustainability issues, they proposed that the government negotiate with suppliers to lower the price of rapid DST and encourage its inclusion in the benefit package of health insurance schemes. 
Box 1

Quotations on the scale-up of rapid DST technologies

We began to use GeneXpert to conduct the screening test for all active TB patients since September, 2017, and now it is free-of-charge for patients, and the provincial-level designated hospital would provide the reagents. (in-depth interview, prefecture-level hospital, Ningxia)

Since September, 2018 all counties in Jilin could conduct molecular DST regularly. 38 of the counties have equipped GeneXpert, and the rest counties used GeneChip or other technologies...(in-depth interview, Jilin academy of TB prevention and treatment)

Every county in our province had equipped GeneXpert, and 73\% of the province had GeneXpert and fluid culture facilities at the same time...GeneXpert testing is free of charge for TB patients. (in-depth interview, CDC, Zhejiang)

GeneXpert has not been covered by the health insurance yet... Now it is provided free of charge during the project period and we are afraid that after the project if patients have to pay out-of-pocket, they would not choose to take the test. (FGD, designated hospital, Zhejiang)

\section{2) Treatment status}

Data from the TBIMS records show that the number DRTB patients included in treatment also increased sharply in all three provinces after the project intervention. The largest increase was in $\mathrm{JL}$, where the number of patients increased nearly 20 -folds from 38 to 747 . The percentage of diagnosed patients in $\mathrm{JL}$ who received treatment rose from $27.0 \%$ to $73.6 \%$. In ZJ and NX, this percentage also increased to $74.7 \%$ and $82.0 \%$ respectively in 2018 (Figure 2).

Among patients who received treatment, the dramatic increase in the proportion of patients who were still under treatment at the end of the six months treatment was also seen in $\mathrm{JL}$, from $55.3 \%$ in 2015 to $92 \%$ in 2018, and the increase was also statistically significant in ZJ, reaching 96.2\% in 2018 (see supplemental table 3 for detailed figures and results of chi-square tests). Results from onsite examination from medical records on this indicator were similar, around $85 \%$ to $90 \%$ in the three provinces (see supplemental table $4)$.

Qualitative findings on the treatment of DRTB patients revealed how project interventions improved the overall quality of DRTB health services. The bi-directional referral policies under which the presumptive DRTB patients are required to be transferred to prefecture-level designated hospital for diagnosis and treatment, have been successfully implemented during the intervention period. Moreover, the supervision on the implementation of these policies by the clinical and public health experts organized by CDC were conducted regularly in all project provinces. These interventions improved physicians' awareness to enroll DRTB patients into standard treatment and helped standardize the clinical practice of health workers 
through regulation and mentoring. The centralized treatment for DRTB patients in prefecture-level designated hospitals also reduced the likelihood of receiving sub-standard treatment in other institutions.

\section{Box 2}

Quotations on the bi-directional referral policies and the supervision on the implementation of interventions

The Bi-directional referral policies have been implemented in around $90 \%$ counties of the provinces. The coordination among the (prefectural-level) infectious disease hospital, county-level TB dispensary and the township hospital was smooth. (in-depth interview, health commission, Jilin)

Our province is small, and the DRTB patients are treated in the provincial level hospital. DRTB patients will be directly transferred from county-level hospital to the provincial level designated hospital. ((indepth interview, health commission, Ningxia)

The CDC would organize a team of experts to come (to check our work) once a month, the Health commission also authorized the Ningxia Fourth Hospital to assess our TB clinical service twice a year, and the results will be reported across the province... (in-depth interview, prefecture-level hospital, Ningxia)

\section{3) The rationality and outcome of treatment}

Results from the TBIMS records showed that the percentage of patients taking a culture test among those under treatment at the end of six months' treatment increased significantly in $\mathrm{JL}$ (from $9.5 \%$ to $40.8 \%$ ) and NX (from 51.5\% to 90.9\%) while the proportion remained the same ZJ (around 75\%) (Figure 3). For JL specifically, the number of DRTB patients taking culture test at the end of the six months of treatment increased from 2 to 280 (see supplemental table 4 for detailed figures and results of chi-square tests). Results from the medical records on this indicator were also similar, except for NX where the sample size was very small $(n=19)$ and may result in the discrepancy in the percentages (see supplemental table 5).

For patients who received a culture test, the negative rate of the sample remained above $90 \%$ in $\mathrm{ZJ}$ and reached $90 \%$ in NX. In JL the percentage reached $70 \%$, still a dramatic improvement from 0 . Results from the medical records showed higher percentage of negative cases $(92.9 \%)$, but the sample size was small. Additionally, medical records examinations found that the percentage of rational regimen was $100.0 \%$, $84.5 \%$, and $77.3 \%$ in $\mathrm{ZJ}, \mathrm{JL}$, and NX respectively. The major problem in the regimen was that the types of drugs were not sufficient, for example, cycloserineoxymycin was often not prescribed when needed.

Qualitative interviews found that the interventions were effective in standardizing physicians' clinical practices and improving the overall quality of DRTB-related clinical services. Nevertheless, challenges remain in further improving the clinical quality. Some physicians complained that certain types of 
second-line anti-TB drugs were restricted by the health insurance, thus they were often not able to prescribe them. Physicians in NX also explained to the experts who examined the medical records that the supply of some second-line drugs in was limited, and often in deficiency. These factors may lead to the insufficient drug type prescribed which was discovered as the major quality problem from the medical records. Additionally, some patients in less developed regions were not willing to go to prefecture-level or higher-level designated hospital for treatment as required, for reasons such as perception of high costs or inconvenience.

\section{Box 3}

Quotations on the challenges to improve quality

We have not received the new formal clinical guideline and thus unable to use it to discuss with the NCMS on the reimbursement policies...Some medicines that could be used by the DRTB patients were restricted by the NCMS, and the physicians would be fined if prescribe those medicines. (in-depth interview, infectious disease hospital, Jilin)

Some DRTB patients were not willing to go to Ningxia Fourth Hospital because it was to faraway for them...(FGD, prefecture-level hospital, Ningxia)

\section{The financial burden of DRTB patients}

\section{1) Patient characteristics}

As shown in table 2, a majority of the patients who participated in the survey were from $\mathrm{JL}$ and $27 \%$ were female, almost the same as of the gender distribution of the registered. The percentages of middle-aged patients and those who resided in the county of registered residence (Hukou) were higher for the survey respondents compared to all registered patients in the three provinces (see supplemental table 1). MDRTB patients were also likely to be the disadvantaged social group as almost $60 \%$ were from rural area, around $2 / 3$ never attending high school, and $1 / 3$ of the patients' household income per capita were below the national poverty line. As for the comparison between patients with or without data on OOP medical expenditure, results show that female and patients with lowest education level were less likely to report OOP. $(P<0.05$, see supplemental table 3$)$. 
Table 2

Characteristics of the participants of the patient survey

\begin{tabular}{|c|c|c|}
\hline & $\mathrm{N}$ & $\%$ \\
\hline Total & 204 & / \\
\hline \multicolumn{3}{|l|}{ Province } \\
\hline ZJ & 39 & 19.1 \\
\hline $\mathrm{JL}$ & 120 & 58.8 \\
\hline NX & 45 & 22.1 \\
\hline \multicolumn{3}{|l|}{ Gender } \\
\hline Female & 55 & 27.0 \\
\hline Male & 149 & 73.0 \\
\hline \multicolumn{3}{|l|}{ Age } \\
\hline$<30$ & 29 & 15.3 \\
\hline $30-59$ & 118 & 62.4 \\
\hline$>59$ & 42 & 22.2 \\
\hline \multicolumn{3}{|l|}{ Ethnicity } \\
\hline Han & 162 & 85.7 \\
\hline Other & 27 & 14.3 \\
\hline \multicolumn{3}{|l|}{ Marriage } \\
\hline Married & 133 & 65.2 \\
\hline \multicolumn{3}{|l|}{ Current place of residence } \\
\hline in the county of registration & 179 & 87.8 \\
\hline in the prefecture of registration, but not the county & 12 & 5.9 \\
\hline in the province of registration, but not the prefecture & 0 & 0.0 \\
\hline outside the province of registration & 13 & 6.4 \\
\hline \multicolumn{3}{|l|}{ Type of registered residence } \\
\hline rural & 121 & 59.3 \\
\hline \multicolumn{3}{|l|}{ Education level } \\
\hline Primary school and below & 66 & 32.4 \\
\hline Junior high school & 66 & 32.4 \\
\hline
\end{tabular}




\begin{tabular}{|c|c|c|}
\hline Senior high school and the equivalent & 41 & 20.1 \\
\hline College and above & 31 & 15.2 \\
\hline \multicolumn{3}{|l|}{ Insurance coverage } \\
\hline UEBMI & 38 & 18.6 \\
\hline NCMS & 93 & 45.6 \\
\hline RBMI & 62 & 30.4 \\
\hline Other & 11 & 5.4 \\
\hline \multicolumn{3}{|l|}{ Household income per capita } \\
\hline Below CNY 3747 (poverty line in 2019) & 67 & 33.5 \\
\hline \multicolumn{3}{|l|}{ NCD status } \\
\hline With other NCD & 76 & 37.3 \\
\hline
\end{tabular}

\section{2) Financial burden in the three provinces and the associated factors}

Results from the patient survey show that mean OOP medical expenditures in ZJ and JL were CNY 25910 and CNY 27816 respectively, which is more than 35\% higher than in NX (table 3). Nevertheless, the mean household income of ZJ patients was $50 \%$ higher than patients in $\mathrm{JL}$, and more than double the income of NX patients. The proportion of patients incurring CHE was also lowest in ZJ, and was similar in $\mathrm{JL}$ and NX. The financial burden of MDRTB patients was still very high, as even using the highest threshold that OOP medical expenditure exceeding $30 \%$ of household income, $77.5 \%$ of the patients incurred $\mathrm{CHE}$.

Table 3

The OOP medical expenditure and the occurrence of CHE for the first six months of treatment

\begin{tabular}{|lllllll|}
\hline Province & $\begin{array}{l}\text { Mean } \\
\text { medical } \\
\text { OOP/CNY }\end{array}$ & $\begin{array}{l}\text { Mean per capita } \\
\text { household income/CNY }\end{array}$ & $\begin{array}{l}\text { Mean household } \\
\text { income/CNY }\end{array}$ & $\begin{array}{l}\% \\
\text { CHE- } \\
10 \%\end{array}$ & $\begin{array}{l}\% \\
\text { CHE- } \\
20 \%\end{array}$ & $\begin{array}{l}\% \\
\text { CHE- } \\
30 \%\end{array}$ \\
\hline ZJ & 25910 & 16614 & 49572 & 77.0 & 68.1 & 62.4 \\
\hline JL & 27816 & 13206 & 32740 & 94.2 & 85.0 & 80.9 \\
\hline NX & 19202 & 5698 & 21691 & 92.8 & 87.9 & 82.6 \\
\hline Total & 24806 & 12181 & 33536 & 90.3 & 82.2 & 77.5 \\
\hline
\end{tabular}


Table 4 shows the results of logistic regression of factors associated with incurring extremely high financial burden for treatment. After adjusting for other demographic and socio-economic factors, patients in $\mathrm{JL}$ were four times more likely to incur extremely high financial burden from treatment than those in ZJ (95\% Cl: 1.01, 16.35). Patients over the age of 59 were much less likely to incur this high financial burden ( $95 \% \mathrm{Cl}: 0.00,0.97)$, probably due to the low willingness to pay for treatment. Patients in the highest income group were less likely to incur high financial burden compared to the poorest group ( $95 \% \mathrm{Cl}: 0.02,0.65)$. Additionally, local patients, (i.e., those that live in the county of registration) have a lower likelihood of incurring extremely high financial burden for treatment, although this is not statistically significant $(\mathrm{OR}=0.14, \mathrm{P}=0.062)$. 
Table 4

Factors associated with incurring extremely high financial burden for DRTB patients

\begin{tabular}{|c|c|c|c|c|}
\hline & OR & $P>t$ & \multicolumn{2}{|c|}{ 95\% Conf. } \\
\hline \multicolumn{5}{|l|}{ Province } \\
\hline ZJ & ref. & & & \\
\hline$J \mathrm{~L}$ & 4.07 & 0.048 & 1.01 & 16.35 \\
\hline $\mathrm{NX}$ & 3.51 & 0.184 & 0.55 & 22.38 \\
\hline \multicolumn{5}{|l|}{ Gender } \\
\hline male & 1.40 & 0.624 & 0.36 & 5.47 \\
\hline \multicolumn{5}{|l|}{ Age } \\
\hline$<30$ & ref. & & & \\
\hline $30-59$ & 0.16 & 0.155 & 0.01 & 2.02 \\
\hline$>59$ & 0.07 & 0.047 & 0.00 & 0.97 \\
\hline \multicolumn{5}{|l|}{ Ethnicity } \\
\hline Han & 2.22 & 0.449 & 0.28 & 17.78 \\
\hline \multicolumn{5}{|l|}{ Marriage } \\
\hline Married & 1.07 & 0.899 & 0.37 & 3.10 \\
\hline \multicolumn{5}{|l|}{ Current place of residence } \\
\hline in the county of registration & 0.14 & 0.062 & 0.02 & 1.11 \\
\hline \multicolumn{5}{|l|}{ Type of registered residence } \\
\hline rural & 0.41 & 0.335 & 0.07 & 2.55 \\
\hline \multicolumn{5}{|l|}{ Education level } \\
\hline primary school and below & ref. & & & \\
\hline junior high school & 0.77 & 0.738 & 0.16 & 3.71 \\
\hline senior high school and above & 0.64 & 0.622 & 0.11 & 3.88 \\
\hline \multicolumn{5}{|l|}{ Insurance coverage } \\
\hline UEBMI & ref. & & & \\
\hline NCMS & 4.91 & 0.109 & 0.70 & 34.59 \\
\hline Other & 3.16 & 0.139 & 0.68 & 14.63 \\
\hline
\end{tabular}




\begin{tabular}{|lllll|}
\hline Average household income & & & & \\
\hline lowest 1/3 & ref. & & & \\
\hline middle 1/3 & 0.41 & 0.330 & 0.07 & 2.54 \\
\hline highest 1/3 & 0.12 & 0.015 & 0.02 & 0.65 \\
\hline NCD status & & & & \\
\hline With other NCD & 0.58 & 0.352 & 0.18 & 1.86 \\
\hline
\end{tabular}

Qualitative findings supported the quantitative results and revealed several possible reasons for the high financial burden, particularly in JL and NX. One reason may be that DRTB patients were treated at prefecture-level designated hospitals or above, usually third-level hospitals, where the reimbursement for public health insurance was relatively low. However, the new financing policies which aimed to limit the percentage of OOP payment as part of the designed interventions, were issued in late 2018 and 2019 in $\mathrm{JL}$ and NX, and may have not been actually implemented in some places at the time of final evaluation. Therefore, in our research, very few patients in JL and NX in our study ever enjoyed the policies. Some interviewees in the less-developed regions also expressed concerns over the availability of additional funds due to financial difficulties of the government. In ZJ there had been some financial assistance policies for DRTB patients to help cover remaining medical expenditures after health insurance reimbursement in 2015.

\section{Box 4}

Quotations on the new financing policies for DRTB patients

For MDRTB patients the reimbursement rate for outpatient visit has been improved to the level of inpatient service in 2015... The health insurance covered $70 \%$ and the provincial and prefectural financing department covered the rest 30\%. (in-depth interview, Zhejiang CDC)

In July 2018 the government began to estimate the money needed for the new financing policies that set the limit of the percentage of the OOP medical expenditure to 10\%. The financing department has passed a plan of 14 million CNY, but the policies had not been implemented yet... (FGD, designated hospital, Jilin)

The financing policies came out late at the prefecture-level mainly due to the financial difficulties of the government that the funds could not be secured, because the economic development in our province was stumbled in recent years... (in-depth interview, Jilin academy of TB prevention and treatment)

The new policies was implemented in Oct. 2018 that the DRTB patients pay less than $30 \%$ of the total medical costs... The patients use their bank card to pay for the medical cost at the hospital, and we transfer the money to patients in 24h...(in-depth interview, health insurance office, Ningxia) 
FGDs with physicians and DRTB patients revealed several reasons for the extremely high financial burden during treatment. One major reason is that some expensive medical services were not covered by the health insurance, and thus not covered by the medical assistance which usually cover the same range of medical services as of the health insurance. Besides, some physicians reported that the cost for medicines not covered by the public health insurance, which were often used in anti-DRTB treatment, should be within $10 \%$ of total costs in their prescriptions as cost control measures regulated by the hospital, and these medicines sometimes went short of supply in the hospital. Consequently, patients were often asked to buy medicines in pharmacies due to physicians' fearing of violating the regulation, or the stock-out problem. Sometimes patients even have to use medical services from private providers, such as intravenous injection of drugs after being discharged from the designated hospital. This may also explain why the officers in ZJ said they provided $100 \%$ coverage, but patients still reported high OOP expenditures. Additionally, medical assistance or supplemental reimbursement were often not directly deducted from the total cost, and patients needed to pay before they could get reimbursement. It was likely that they still had not received medical assistance, or forget about the assistance when reporting OOP medical expenditures.

Moreover, DRTB patients often had co-morbidities. These costs could not be separated from TB treatment when patients report expenditures, but the treatment costs for co-morbidities were not eligible for additional reimbursement or assistance. Some patients also admitted that they defaulted treatment due to financial difficulties. 
Box 5

Quotations on the financial burden of DRTB patients

I was hospitalized in Ningxia Fourth hospital last year. I stayed in hospital for 90 days and had taken five times of fiberoptic bronchoscopy... I was enrolled in URBMI and paid over CNY 20,000 out-ofpocket. I am a farmer and I borrowed money to pay for the cost...(FGD, DRTB patient in Ningxia)

Currently I do not need to pay out-of-pocket under the special policy for outpatient visit, but I was once hospitalized for liver damage, and I paid CNY 40-50,000 out-of-pocket for this hospitalization, and the actual reimbursement rate was only $60 \%$...(FGD, DRTB patient in Zhejiang)

I experienced shortage of medicines in hospitals, and I felt that the side-effects of domestic medicines were substantial and I bought medicines made in India... When I was hospitalized, some medicines were not covered by health insurance and they cost several thousands CNY... (FGD, DRTB patient in Zhejiang)

I was hospitalized for 6 to 9 months in total, and I needed to pay over 100, 000 CNY before I could get reimbursed... Sometimes there was a shortage of medicines in the hospital, I needed to go the pharmacies to buy medicines which were more expensive... The auxiliary medicines could were not covered by health insurance (FGD, DRTB patients in Jilin)

I needed to go to private clinic for injection as the medicine was prescribed by the designated hospital, not the community health care centers, because the community health care centers would not help you inject the medicines not prescribed by them. I paid the private clinic 20 CNY each time for the injection...(FGD, DRTB patient in Jilin)

I used up my money during hospitalization, and I did not take medicines after discharging from hospital... (FGD, DRTB patient in NX)

\section{Discussions}

Our results show that the quality of clinical services for screening, diagnosis, and treatment of DRTB patients significantly improved during the intervention period. Gains were especially large for JL where there was almost no standardized DRTB treatment and management prior to project implementation. However, the financial burden of DRTB patients was not remedied during the intervention period, likely due to the delays in implementing DRTB financing policies.

\section{Effective interventions and further challenges in improving the quality of DRTB clinical services}

Our study findings align with existing research regarding the effectiveness of rapid DST technologies in improving DRTB case finding capacity. It is also notable that the supporting policies to guarantee the 
supply of reagents for assays and provide tests free-of-charge for patients have ensured the use of the new technologies in the screening for DRTB patients upon necessity. The shortened diagnosis timeline and strict referral policies also increased the treatment inclusion rate, as evidenced by other studies [5, 16]. Although the price for the GeneXpert test is still expensive and not covered by the health insurance, the government must ensure the sustainability of funds to maintain or further improve case detection capacity after project ends. The study on the use of GeneXpert in Ghana also mentioned about the concern of government budget to run GeneXpert [33]. As physicians suggested during the interviews, the health insurance office could also negotiate with the reagent producers to lower the price of testing, and thus providing coverage for rapid DST at a relatively lower cost. For other low- and middle- income countries with high burden of TB, the high demand for testing reagents and the pooled purchase by the government could potentially lower the unit price and make it affordable for the government to cover the testing cost.

While treatment enrollment and adherence largely improved across the provinces, $20 \%-30 \%$ of patients did not initiate standard treatment. Additionally, $25 \%$ of the patients in $\mathrm{ZJ}$ and $60 \%$ in $\mathrm{JL}$ did not take a culture test at the end of six months' treatment as required by the clinical guidelines. Although the bidirectional referral policies and the supervision on the clinical practice of physicians may regulate health providers' behaviors, the interventions aiming at encouraging DRTB patients to follow standard treatment were nearly nonexistent. Additionally, the contradictions between the clinical needs of DRTB treatment and the public health insurance regulations regarding the prescription of medicines should be resolved to ensure treatment quality. The shortage of second-line drug supply in less-developed regions also needs to be taken seriously and addressed through policy intervention.

\section{Challenges and potential policies to relieve the financial burden of DRTB patients}

The universal occurrence of the CHE among the DRTB patients indicates that financial burden is still heavy under the current health insurance and medical assistance system in China. Failure to address this issue may become a bottleneck for further improving the quality of DRTB-related health services and treatment outcomes. The fact that the new financing policies were not implemented on time indicates the difficulties in mobilizing financial resources to relieve the economic burden of patients, especially for the less-developed regions with relatively low government revenue and tight health insurance budgets. In recent years the Chinese government has focused on the national policy of targeted poverty-alleviation [34], and in our study the poorest $1 / 3$ patients had much higher likelihood of incurring extremely high financial burden during treatment, which would in turn aggravate their poverty status. The specialized funding to support DRTB patients to complete treatment, especially for those poorest patients, could potentially be secured through the national targeted poverty alleviation program. Such a program should provide coverage for DRTB as well as comorbidities, which were extremely burdensome for DRTB patients. Additionally, as migrant patients may also have more difficulties accessing DRTB care and bear higher financial burden of treatment, though not explored in detail in this study but revealed in other 
studies [35-37], the assistance polices for DRTB policies may also cover migrant workers who are often excluded from social assistance policies without local registered residence (Hukou).

In addition to financing policies, cost control is also crucial for reducing the financial burden and developing feasible financial support policies for DRTB patients. On the one hand, the unit-price of some tests and medicines are expensive, as evidenced in previous studies and our qualitative research [13]. The health insurance office may take the lead in the negotiation to lower the price for the necessary tests and medicines, as suggested before for the promotion of GeneXpert. On the other hand, the clinical practices need to be further standardized and avoid the providing unnecessary services due to profit-driven motivations, which has been widely discussed during the healthcare reform in China [38-40]. Our findings that patients in JL paid the highest OOP during the first 6 months and had the highest likelihood of incurring catastrophic expenditure could possibly be associated such motivations. Our qualitative research with directors and physicians from infectious disease hospitals and TB dispensaries in JL did reveal their concerns about the revenue from medical services which is related to their income. Physicians in the infectious department in general hospitals in other provinces also said that their department did not earn as much revenue as other departments, but there were some mechanisms to balance the income of physicians in the hospital. Nevertheless, the infectious disease hospitals or TB dispensaries in JL do not have such departments that usually generate higher income. In light of these findings, the motivations to earn income from medical services need to be altered for better control of DRTB patients' financial burden. The case-based payment approach which sets a fixed payment amount for a single case based on a reasonable estimation of the appropriate treatment cost, could be a possible solution [41]. This is particularly important for governments in low- and middle-income countries with tight budgets to provide universal coverage for DRTB patients.

Although our findings provide useful policy implications, there are several limitations. First, the sample size for the examination of medical records examination is very small, and the representativeness of the sample could not be well-proved. Additionally, we only collected information on the treatment experiences and OOP for the first six months of treatment given feasibility constraints. Therefore, we did not examine medical expenditures for the whole treatment course. Recall bias was likely to happen as the many patients reported their experiences more than one year ago. Moreover, compared to the overall registered patients, the sample for patient survey included a lower percentage of migrant patients, and the financial burden may be under estimated for the overall patient group. Despite these limitations, we believe our findings on the quality of DRTB related clinical services are still sound, as results from medical records are similar to the those from registration data. The finding that heavy financial burden was still prevailing among DRTB patients were also unlikely to be influenced by the limitations with our rich evidence.

With regards to the challenges and potential strategies we identified in further improving the clinical quality and relieving the financial burden of DRTB patients, future projects may design interventions on key aspects such as promoting the optimal use of new DST technologies through sustainable funding, standardizing the clinical practices of physicians as well as developing impelling policies to provide 
financial protection and control treatment cost for DRTB patients, and evaluate the effectiveness of these interventions based on concrete evidence.

\section{Conclusions}

The quality of DRTB related clinical services has significantly improved since the implementation of comprehensive interventions on the promotion of rapid DST technology and the regulation of clinical practices. Nevertheless, the high financial burden of DRTB patients have not been alleviated, primarily due to the delayed implementation of financing policies. Stronger political commitment and leadership are required to mobilize multiple financing resources to provide additional financial support to DRTB patients.

\section{Abbreviations}

DRTB: drug-resistant tuberculosis

WHO: World Health Organization

RRTB: rifampicin-resistant tuberculosis

MDRTB; multi-drug resistant tuberculosis

UEBMI: urban employee basic medical insurance

URBMI: urban resident basic medical insurance

NCMS: new cooperative medical schemes

DST: drug susceptibility test

OOP: out-of-pocket

NHC: National Health Commission

\section{ZJ: Zhejiang Province}

JL; Jilin Province

NX: Ningxia Hui Autonomous Region

TBIMS: Tuberculosis Information Management System

FGD: focus-group discussion

CDC: Center for Disease Control 


\section{Declarations}

\section{Ethics approval and consent to participate}

The implementation of China NHC-Gates TB Phase III project has received the ethical approval from China CDC (No.201626). The protocol of the overall study design and data collection tools was reviewed and approved by the Institutional Review Board of Duke University (IRB Approval code: 2017-0768). Consent form was obtained before individual data collection and could be provided upon request.

\section{Consent for publication}

Consent form was obtained before individual data collection and could be provided upon request.

\section{Availability of data and materials}

The datasets generated and analyzed during the current study are not publicly available due to the regulations of China CDC. Readers of the article need to discuss with China CDC and obtain their permission before the release of the dataset.

\section{Competing interests}

All authors declare no competing interests.

\section{Funding}

The study was funded by the Bill and Melinda Gates Foundation.

\section{Authors' contributions}

Weixi Jiang conducted literature search. Shenglan Tang, Weixi Jiang, Qi Zhao and Mengqiu Gao designed the study. Qi Zhao, Mengqiu Gao, Zhipeng Li, Fei Huang and Ni Wang collected the data and helped interpret the data. Weixi Jiang and Zhipeng Li analyzed the data. Weixi Jiang prepared the manuscript; Qi Zhao, Qian Long, Weibing Wang, Ni Wang and Shenglan Tang revised the manuscript and provided valuable suggestions to improve the manuscript.

\section{Acknowledgements}


Dr Xiexiu Wang, Dr Shuigao Jin, Dr. Shiwen Jiang, Dr. Lixia Wang and Dr. Lusheng Wang, the five senior advisors of the project, provided very helpful technical support to the project, to which the authors of the paper are immensely grateful. The authors of the paper would also express their sincere appreciation to Dr. Daniel Chin and Shitong Huan from the Gates Foundation, and other colleagues from China CDC at national, provincial, prefectural and county levels, as well as from TB designated hospitals at prefectural and county levels in the three project provinces who generously contributed their time to the implementation of the study. The authors also would like thank Dr. Kaci Kennedy McDade for language editing and valuable suggestions.

\section{References}

1. Abubakar I, Zignol M, Falzon D, Raviglione M, Ditiu L, Masham S, et al. Drug-resistant tuberculosis: Time for visionary political leadership. Lancet Infect Dis. 2013;13(6):529-39. Available from: http://dx.doi.org/10.1016/S1473-3099(13)70030-6

2. World Health Organization. Global Tuberculosis Report 2020. 2020.

3. World Health Organization. Available from: https://www.who.int/sdg/targets/en/. Accessed on 2020 Aug 13.

4. Oo T, Kyaw KWY, Soe KT, Saw S, Satyanarayana S, Aung ST. Magnitude and reasons for prediagnosis attrition among presumptive multi-drug resistant tuberculosis patients in Bago Region, Myanmar: A mixed methods study. Sci Rep. 2019;9(1):1-10. Available from: http://dx.doi.org/10.1038/s41598-019-43562-3

5. Shewade D, Kokane AM, Singh AR, Verma M, Parmar M, Chauhan A, et al. High pre-diagnosis attrition among patients with presumptive MDR-TB: an operational research from Bhopal district, India. BMC Health Serv Res. 2017;17(1):1-10.

6. Boyd R, Ford N, Padgen P, Cox H. Time to treatment for rifampicin-resistant tuberculosis: Systematic review and meta-analysis. Int J Tuberc Lung Dis. 2017;21(11):1173-80.

7. Pablos-Méndez A, Gowda DK, Frieden TR. Controlling multidrug-resistant tuberculosis and access to expensive drugs: A rational framework. Bull World Health Organ. 2002;80(6):489-95.

8. Falzon D, Jaramillo E, Wares F, Zignol M, Floyd K, Raviglione MC. Universal access to care for multidrug-resistant tuberculosis: An analysis of surveillance data. Lancet Infect Dis. 2013;13(8):6907. Available from: http://dx.doi.org/10.1016/S1473-3099(13)70130-0

9. Glasziou P, Straus S, Brownlee S, Trevena L, Dans L, Guyatt G, et al. Evidence for underuse of effective medical services around the world. Lancet. 2017;390(10090):169-77.

10. Tunçalp Ö, Were WM, MacLennan C, Oladapo OT, Gülmezoglu AM, Bahl R, et al. Quality of care for pregnant women and newborns-the WHO vision. BJOG An Int J Obstet Gynaecol. 2015;122(8):1045-9.

11. Tang $S$, Wang $L$, Wang $H$, Chin DP. Access to and affordability of healthcare for TB patients in China: Issues and challenges. Infect Dis Poverty. 2016;5(1):1-5. Available from: 
http://dx.doi.org/10.1186/s40249-016-0096-y

12. Pan H-Q, Bele S, Feng Y, Qiu S-S, Lü J-Q, Tang S-W, et al. Analysis of the economic burden of diagnosis and treatment of tuberculosis patients in rural China. Int $\mathrm{J}$ Tuberc Lung Dis. 2013;17(12):1575-80.

13. Zhang Y, Liu X, Yang L, Zhang G, Gu Z, Chen Z, et al. Barriers and strategies: A review of access to affordable multi-drug resistant tuberculosis medication in China. Infect Drug Resist. 2020;13:367987.

14. Xu ZH, Xiao T, Li YH, Yang KY, Tang Y, Bai LQ. Reasons for Non-Enrollment in Treatment among MultiDrug Resistant Tuberculosis Patients in Hunan Province, China. PLoS One. 2017;12(1):e0170718.

15. Wang Y, McNeil EB, Huang Z, Chen L, Lu X, Wang C, et al. Household financial burden among multidrug-resistant tuberculosis patients in Guizhou province, China: A cross-sectional study. Vol. 99, Medicine (Baltimore). Lippincott Williams \& Wilkins, WK Health; 2020. p. e21023.

16. Xu C, Li R, Shewade HD, Jeyashree K, Ruan Y, Zhang C, et al. Attrition and delays before treatment initiation among patients with MDR-TB in China (2006-13): Magnitude and risk factors. PLoS One. 2019;14(4):1-16.

17. Hutchison C, Khan MS, Yoong J, Lin X, Coker RJ. Financial barriers and coping strategies: a qualitative study of accessing multidrug-resistant tuberculosis and tuberculosis care in Yunnan, China. BMC Public Health. 2017;17(1):1-11.

18. World Health Organization; Available from: https://www.who.int/tb/country/data/profiles/en/, Accessed on 2020 Aug 13

19. Chen J, Peng P, Du Y, Ren Y, Chen L, Rao Y, et al. Early detection of multidrug- and pre-extensively drug-resistant tuberculosis from smear-positive sputum by direct sequencing. BMC Infect Dis. 2017;17(1):1-7.

20. Pang Y, Xia H, Zhang Z, Li J, Dong Y, Li Q, et al. Multicenter Evaluation of Genechip for Detection of Multidrug- Resistant Mycobacterium tuberculosis. 2013;51(6):1707-13.

21. Atashi S, Izadi B, Jalilian S, Madani SH, Farahani A, Mohajeri P. Evaluation of GeneXpert MTB / RIF for determination of rifampicin resistance among new tuberculosis cases in west and northwest Iran. New Microbes New Infect. 2017;19:117-20. Available from:

http://dx.doi.org/10.1016/j.nmni.2017.07.002

22. Christopher PM, Widysanto A. GeneXpert Mycobacterium tuberculosis / Rifampicin Assay for Molecular Epidemiology of Rifampicin-Resistant Mycobacterium tuberculosis in an Urban Setting of Banten Province, Indonesia. 2019;351-8.

23. Yin J, Yuan J, Hu Y, Wei X. Association between directly observed therapy and treatment outcomes in multidrug-resistant tuberculosis: A systematic review and meta-analysis. PLoS One. 2016;11(3):114.

24. Alipanah N, Jarlsberg L, Miller C, Linh NN, Falzon D, Jaramillo E, et al. Adherence interventions and outcomes of tuberculosis treatment: A systematic review and meta-analysis of trials and observational studies. Vol. 15, PLoS Medicine. 2018. 1-44 p. 
25. Richter LM, Lönnroth K, Desmond C, Jackson R, Jaramillo E, Weil D. Economic support to patients in HIV and TB grants in Rounds 7 and 10 from the Global Fund to Fight AIDS, Tuberculosis and Malaria. PLoS One. 2014;9(1).

26. Li R, Ruan Y, Sun Q, Wang X, Chen M, Zhang H, et al. Effect of a comprehensive programme to provide universal access to care for sputum-smear-positive multidrug-resistant tuberculosis in China: A before-and-after study. Lancet Glob Heal. 2015;3(4):e217-28. Available from: http://dx.doi.org/10.1016/S2214-109X(15)70021-5

27. Baral SC, Aryal Y, Bhattrai R, King R, Newell JN. The importance of providing counselling and financial support to patients receiving treatment for multi-drug resistant TB: Mixed method qualitative and pilot intervention studies. BMC Public Health. 2014;14(1).

28. Tang S. Tackling challenges of TB/MDRTB in China: Concerted actions are imperative. Infect Dis Poverty. 2015;4(1):1-3.

29. China National Bureau of Statistics. Available from: http://www.stats.gov.cn/tjsj/ndsj/2019/indexch.htm. Accessed on 2020 Aug 17.

30. Kenward MG, Carpenter J. Multiple imputation: Current perspectives. Stat Methods Med Res. 2007;16(3):199-218.

31. Wingfield T, Boccia D, Tovar M, Gavino A, Zevallos K, Montoya R, et al. Defining Catastrophic Costs and Comparing Their Importance for Adverse Tuberculosis Outcome with Multi-Drug Resistance: A Prospective Cohort Study, Peru. PLoS Med. 2014;11(7).

32. Ritchie J, Spencer L, O'Connor W. Carrying out qualitative analysis. In: Ritchie J, Lewis J, editors. In origins of qualitative research practice: A guide for social science students and researchers. SAGE Publications; 2003. p. 219-62.

33. Boakye-appiah JK, Steinmetz AR, Pupulampu P, Ofori-yirenkyi S, Tetteh I, Frimpong M, et al. High prevalence of multidrug-resistant tuberculosis among patients with rifampicin resistance using GeneXpert Mycobacterium tuberculosis/rifampicin in Ghana. Int J Mycobacteriology. 2016;5(2):22630. Available from: http://dx.doi.org/10.1016/j.jimyco.2016.02.004

34. Dai X, Wang L, Ren Y. The Effects of China's Targeted Poverty Alleviation Policy on the Health and Health Equity of Rural Poor Residents: Evidence from Shaanxi Province. Healthcare. 2020;8(3):256.

35. Nellums LB, Rustage K, Hargreaves S, Friedland JS. Multidrug-resistant tuberculosis treatment adherence in migrants: A systematic review and meta-analysis. BMC Med. 2018;16(1):1-11.

36. Bele $S$, Jiang W, Lu H, You H, Fan H, Huang L, et al. Population aging and migrant workers: Bottlenecks in tuberculosis control in rural China. PLoS One. 2014;9(2).

37. Long Q, Li Y, Wang Y, Yue Y, Tang C, Tang S, et al. Barriers to accessing TB diagnosis for rural-tourban migrants with chronic cough in Chongqing, China: A mixed methods study. BMC Health Serv Res. 2008;8:1-10.

38. Yip Dr WC-M, Hsiao Prof W, Meng Prof Q, Chen Prof W, Sun PhD X. Realignment of incentives for health-care providers in China. Lancet, 2010;375(9720):1120-30. 
39. Ramesh M, Wu X. Health policy reform in China: Lessons from Asia. Soc Sci Med. 2009;68(12):2256-62.

40. Long Q, Qu Y, Lucas H. Drug-resistant tuberculosis control in China: progress and challenges. Infect Dis Poverty. 2016;5(1):9.

41. Gao C, Xu F, Liu GG. Payment reform and changes in health care in China. Soc Sci Med. 2014;111(pp 10-16):10-6.

\section{Figures}

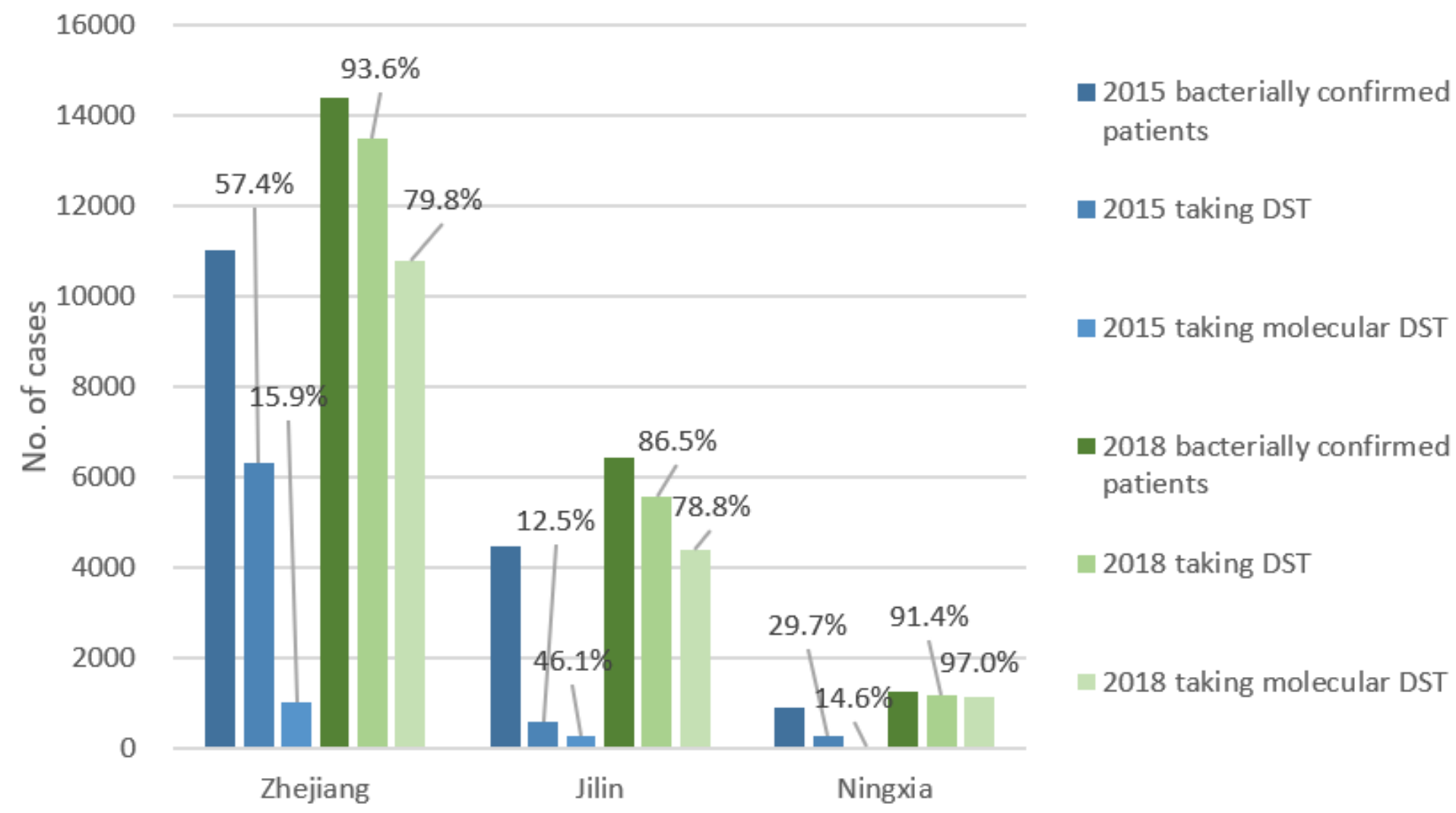

\section{Figure 1}

The number and percentage of bacterially confirmed patients taking DST at baseline and final evaluation 


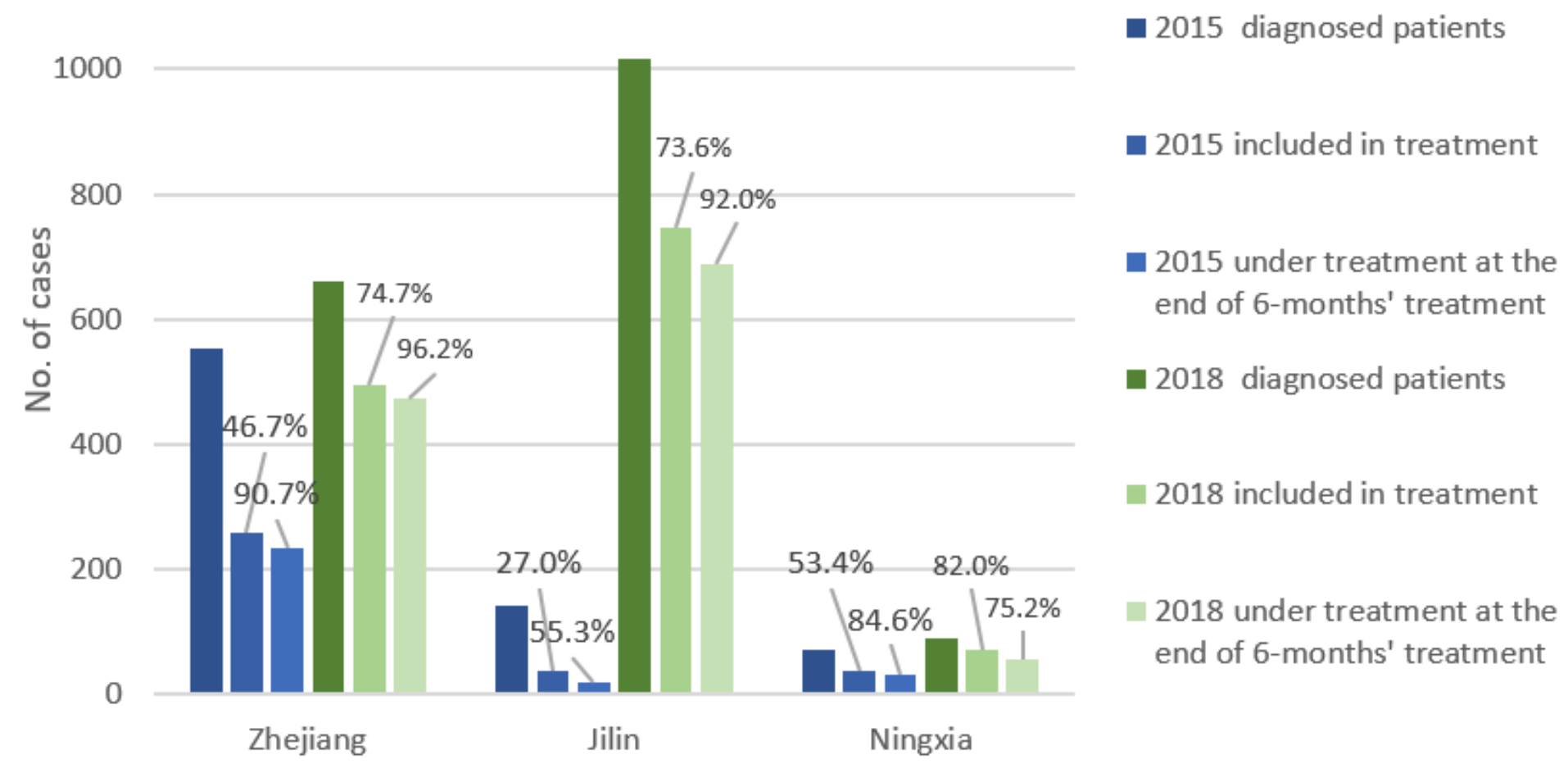

Figure 2

DRTB patients diagnosed, included in treatment and under treatment at the end of 6-months' treatment 


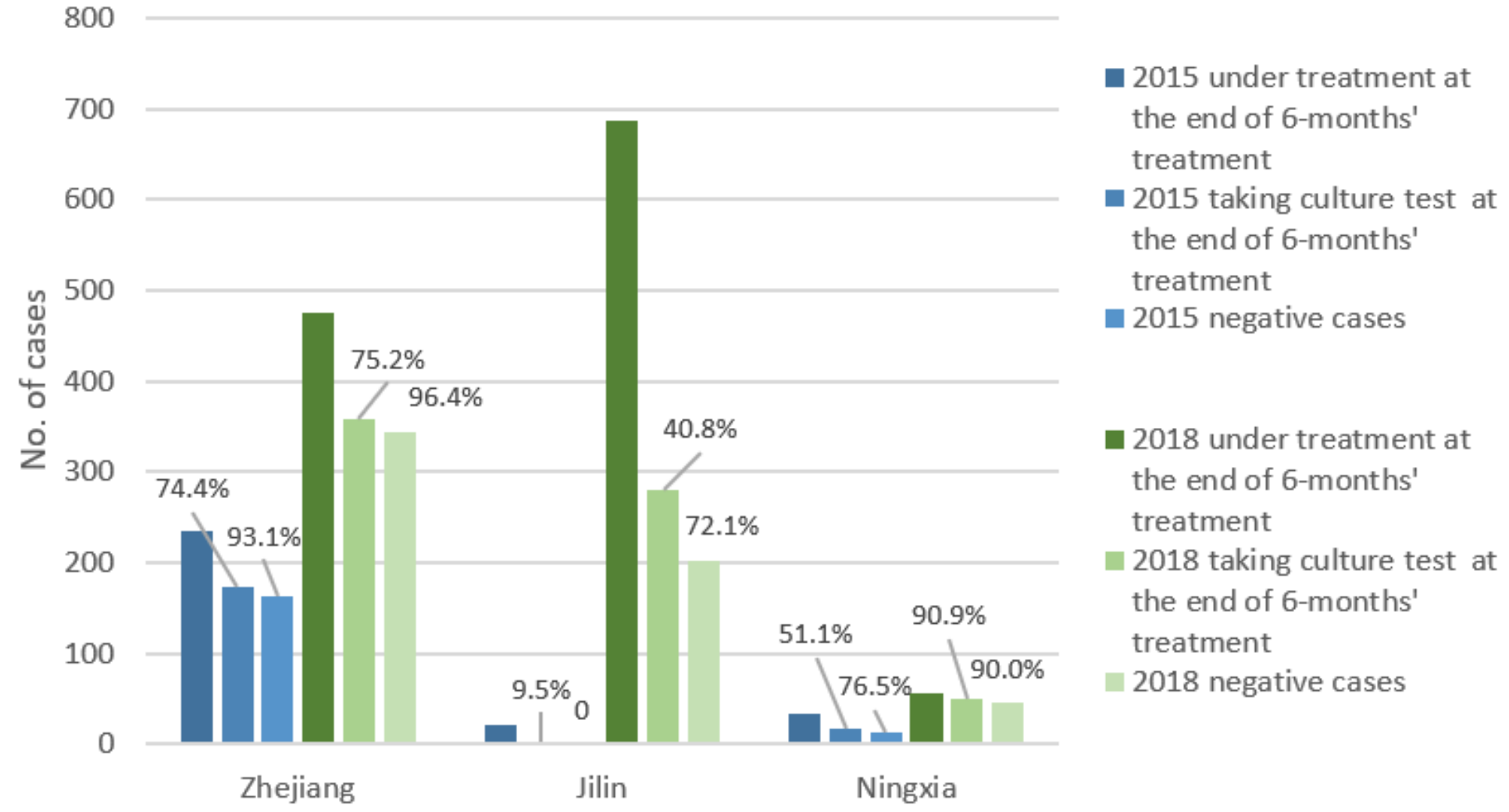

Figure 3

DRTB patient taking culture test at the end of 6-months' treatment and the negative cases

\section{Supplementary Files}

This is a list of supplementary files associated with this preprint. Click to download.

- SupplementalMaterials.docx 